\title{
LA TRAYECTORIA LABORAL DE LAS MUJERES CANARIAS EN LAS CUATRO ÚLTIMAS DÉCADAS
}

\author{
$M^{a}$ del Carmen Barrera Casañas \\ Universidad La Laguna \\ cbarrera@ull.es
}

Recibido: 20-02-09

Aceptado: 24-02-09

\section{Resumen}

En este artículo se estudia la trayectoria laboral de las mujeres canarias en las últimas cuatro décadas. Se atiende a las diferencias de género y se hace un análisis comparativo con la situación laboral del resto de las mujeres españolas. Todo esto resulta novedoso, especialmente si consideramos que el comportamiento laboral de las canarias ha permanecido excluido de las investigaciones sobre la situación laboral de las españolas. El artículo también refleja hasta qué punto el proceso de modernización experimentado en al archipiélago canario ha incidido sobre las pautas laborales de las mujeres, y cómo su incidencia se ha manifestado en los comportamientos entre las diferentes generaciones.

Palabras clave: mujeres, género, trabajo, actividad económica, diferencias, oportunidades, edad, estado civil, estudios.

\begin{abstract}
This article studies the labour path of Canary Island women in the last four decades. It presents the results of differences between genders and makes a comparing analysis of the labour situation on the remainder of Spanish women. All of this is brand new,
\end{abstract}


especially if we considerer that the working behaviour of canary women had been excluded from the researches about the labour situation of the Spanish women in general. Moreover, the article reflects how effective was the process of modernisation experienced in the Canary Islands in influence the working behaviour of women and how these effects appeared in the behaviour of different generations.

Key words: women, gender, work, economic activity, differences, opportunity, age, marital status, studies.

\section{Introducción}

En los últimos años han aumentado considerablemente las publicaciones sobre la situación laboral de las mujeres. En España, debido principalmente a las condiciones sociopolíticas, estas investigaciones comenzaron a desarrollarse y a salir a la luz con mayor retraso que en el resto de los países europeos, cobrando una gran fuerza a partir de los años ochenta del pasado siglo, especialmente desde ciertos ámbitos disciplinares (Sociología, Antropología, Historia, Economía). Desde la Sociología, las publicaciones precursoras en este tema se han extendido por todo el Estado. Sin embargo, en lo que respecta a la comunidad canaria todavía son escasos los estudios científicos en los que se ha considerado la situación laboral de las mujeres. Precisamente este artículo recoge algunas de las conclusiones más relevantes sobre el comportamiento laboral de las mujeres canarias ${ }^{1}$. En este artículo se estudia la trayectoria laboral de las canarias en las cuatro últimas décadas, desde 1977 hasta 2007, atendiendo a la trayectoria laboral.

Las conclusiones aquí presentadas resultan del vaciado, explotación y análisis de las Encuestas de Población Activa - segundos trimestres - desde 1977 hasta 2007. Se ha atendido a la evolución de la actividad, ocupación, paro, situación profesional y

\footnotetext{
${ }^{1}$ Fruto de la primera tesis doctoral defendida en Canarias, y originariamente titulada Trabajo, educación y familia de las mujeres canarias. Análisis sociológico del papel de las mujeres en los últimos 20 años.
} 
ocupacional, atendiendo a las diferencias de género. Ello se ha llevado a cabo a través del análisis transversal ${ }^{2}$ y longitudinal ${ }^{3}$. Asimismo, se ha realizado un análisis comparativo entre la trayectoria laboral de las mujeres canarias con la del resto de las españolas. Por otro lado, los resultados desprendidos de la situación laboral de las canarias en las islas del Archipiélago obedecen a la explotación de los Censos de Población de 1981, 1991 y 2001, así como de la Encuesta de Población de Canarias de 1996.

Las conclusiones sobre algunos cambios experimentados en el calendario matrimonial y reproductivo de las mujeres, sobre los que también se harán referencia, se desprenden de la explotación de Censos de Población 1970, 1981, 1991 y 2001, la Encuesta de Población de Canarias de 1996 y los Movimientos Naturales de Población Española desde 1977 hasta 2007, relativos a Canarias y ámbito estatal.

Paralelamente al propósito de indagar en la trayectoria laboral de las mujeres canarias en los últimos cuarenta años, en el artículo se muestra también cómo el proceso de modernización experimentado en el archipiélago canario ha incidido sobre las pautas laborales de las mujeres y hasta qué punto su incidencia se ha reflejado en los diferentes comportamientos entre las diversas generaciones. Los resultados de ambos análisis se han encaminado, por un lado, al esclarecimiento de la trayectoria participativa de las mujeres en la producción extradoméstica y de su posición en la división sexual del trabajo en la sociedad canaria. Por otro lado, el estudio de la trayectoria laboral de las canarias también ha permitido obtener un mayor conocimiento sobre la realidad de la sociedad canaria en su conjunto.

\footnotetext{
${ }^{2}$ Segmentación social por clases de edad.

${ }^{3}$ Segmentación social por cohortes generacionales.
} 


\section{La trayectoria laboral de las mujeres canarias en las cuatro últimas décadas}

Pese a las peculiaridades demográficas y económicas que presenta el archipiélago canario, los procesos de cambio social experimentados en las últimas cuatro décadas han afectado a las canarias de forma similar que al resto de las españolas (Barrera, 2007). Si bien estos cambios han incidido sobre las nuevas pautas sociales de las mujeres, éstos también han sido determinados por los diferentes comportamientos del colectivo femenino, especialmente por las cohortes más jóvenes y más concretamente por aquellas que han podido invertir más tiempo en formación y estudios (Alberdi, Escario y Matas, 2002; Aguilar, 2001; Montañes y Olivari, 2000; Torns, 2000). De este modo hemos podido observar claramente, que las transformaciones experimentadas en las pautas laborales de las mujeres canarias en las cuatro últimas décadas han obedecido principalmente a las estrategias utilizadas por éstas (de forma consciente e inconsciente) en la mayor inversión en formación.

A pesar de los cambios sustanciales que ha experimentado el mercado de trabajo en los últimos años (Segura, 2001), la creciente inversión en formación por parte de las mujeres ha posibilitado su mayor relación con el trabajo extradoméstico, así como una mejora de su estatus en lo que respecta a su mayor autonomía y posición social (Beck-Gersheim, Butler y Puigvert, 2001; Suárez, 2004). Además, ello ha comenzado a debilitar los cimientos sobre los que se sustenta el sistema patriarcal.

Huelga decir, que las pautas laborales de las mujeres deben de ser interpretadas desde los fenómenos socioculturales, estimando, entre otra serie de factores, el nivel de formación, la situación familiar, el papel desempeñado en el ámbito doméstico (trabajo del hogar y familia), los fenómenos culturales (actitudes y conducta hacia el trabajo extradoméstico), la propia estructura del mercado de trabajo y los tipos de ofertas de empleo. En las cuatro últimas décadas, la incidencia de todos estos factores no sólo se ha reflejado en las relaciones de género, sino también entre las cohortes generacionales, así como entre las mujeres de los diversos ámbitos geográficos (Barrera, 2001; Castaño, 2004; Mosteiro, 2001). Así, históricamente las canarias han tendido a contraer 
matrimonio antes que el resto de las españolas, han tenido un mayor número de hijos/as y por lo tanto mayores responsabilidades y cargas familiares. Además, hasta hace poco tiempo, el nivel de estudios de las primeras ha sido inferior al de las segundas. Estas son algunas de las características que han condicionado las pautas laborales de las canarias con respecto al resto de las españolas. No obstante, y a pesar de la incidencia de todos estos factores, especialmente desde mediados de los 80, la creciente incorporación de las canarias al mercado de trabajo ha sido más que evidente. Esto ha resultado ser parte de la expresión del rechazo que, de manera masiva, comenzaron a mantener las mujeres al desempeñar trabajos no remunerados (en situación de ayuda familiar y trabajadoras del hogar o "amas de casa"), que eran más frecuentes hasta finales de la década de los 80. También evidencia la renuncia de las mujeres a trabajar en la economía sumergida, donde históricamente han tenido un peso importante. Todo ello ha provocado cambios sustanciales en los comportamientos laborales por cohortes generacionales.

El fenómeno de la inserción "masiva" de las mujeres canarias se produjo más tardíamente que entre el resto de las españolas. Esta tuvo lugar en un período de recesión económica, que se produjo en la comunidad canaria entre 1981-1985, y que fue impulsada posteriormente por una etapa de recuperación económica (1985-1988). Durante estos años se incorporaron a trabajar las cohortes nacidas durante el boom de la natalidad, que había tenido lugar en la primera mitad de los 70. Las mujeres vieron un momento muy propicio para buscar empleo, ya que en Canarias fue especialmente durante estos años cuando se produjo la creación de empleo en el sector servicios.

En años anteriores, y coincidiendo con un período de crisis que tuvo lugar en el archipiélago canario entre 1977-1981, las mujeres habían ganado actividad y ocupación, pero éstas apenas representaba una cuarta parte sobre el total del colectivo femenino. Durante esta etapa, el crecimiento de la actividad se había visto motivado por la creación de empleo (especialmente en el mercado secundario), así como por el desempleo, mayoritariamente por las mujeres que se integraban por primera vez en el mercado laboral. El paro femenino de los años 70 presentaba cierta similitud con el de la década de los 60, esto es, se caracterizaba por su bajo índice, que respondía a la 
escasa relación que mantenían las mujeres con el mercado de trabajo. Por ello, este tipo de paro llegó a ser considerado como un factor "positivo", en la medida en que hacía referencia a una mayor relación de las mujeres con la actividad económica (Barrera, 2004).

Hasta principios de los 80, el número de canarias activas y empleadas tendía a situarse por debajo del resto de las españolas. En estos años, el máximo de la actividad y ocupación femenina se producía entre los 20-24 años. Debido a que las canarias invertían menos tiempo en los estudios que el resto de las españolas, estas comenzaban a trabajar más pronto. No obstante, a partir de los 24 años se apreciaba un descenso de su actividad y empleo, ocasionado por el matrimonio a edades tempranas, el trabajo del hogar y la crianza de los hijos, fenómeno que a estas edades era más común entre las canarias que entre el resto de las españolas.

Durante los primeros años de la década de los 80 , la competencia laboral entre las generaciones femeninas comienza a ser evidente. Las mayores (con más de 50 años) y ancianas (edades cercanas a la retirada laboral) empezaron a perder más empleo que las jóvenes (menores 30 años) y adultas (30-50 años), y eso que durante los años anteriores, especialmente en las etapas de baja económica, los dos primeros colectivos habían abandonado menos su puesto de trabajo. La mayoría de las estas mujeres desempeñaban actividades que ni siquiera los varones se atrevían a aceptar en los momentos más acentuados de la crisis (tal es el caso de las actividades de "limpieza", "saneamiento", "servicio doméstico"). Debido a ello, y a la propia segregación sexual del trabajo, las mujeres "protegieron” su empleo (Durán, 1992). La competencia por estos puestos de trabajo era más obvia entre las cohortes femeninas que entre los géneros.

Muchas de las canarias que habían comenzado a integrarse laboralmente a principios de los 80, una vez superaron la barrera del primer empleo, tendieron a comportarse como mano de obra "primaria", permaneciendo en el mercado, incluso, en situación de desempleo. A mediados de los 80, y motivado por el impulso de la recuperación económica (1985-1988), las canarias continuaron ganando ocupación. 
Debido a la mayor inversión en los estudios, la inserción laboral continuaba produciéndose a edades más tardías (Barrera, 2005). En años anteriores, este fenómeno resultaba ser más visible entre el resto de las españolas que entre las canarias, pero a finales de los 80 estas pautas comienzan a asemejarse.

A principios de los 90 la resistencia de las mujeres a abandonar el mercado de trabajo resulta más que evidente, especialmente por parte de las canarias más formadas. Debido a ello, y a la progresiva inserción laboral de las mujeres, las tasas de paro se incrementaron considerablemente. Este aumento fue tan considerable que, casi en la segunda mitad de los 90, en Canarias las jóvenes paradas comenzaron a superar numéricamente a los jóvenes parados. Fenómeno que, dicho sea de paso, ya venía produciéndose en el ámbito nacional desde principios de los 90 (Ayllon, 1995). Desde los año 90 hasta la actualidad, tanto en el Archipiélago Canario como en el resto del país, el número de paradas no ha dejado se situarse por encima del número de parados ${ }^{4}$.

Con motivo de la mayor inversión en los estudios, y la consiguiente posposición laboral, la máxima de la actividad y del empleo femenino, que se apreciaba en los años 80, se extendió, en la década de los 90, al grupo de 25-29 años. En general comienza a observarse un desplazamiento de la disminución de la desigualdad, tanto de la actividad como de la ocupación, hacia la derecha de la curva de los grupos de edad, esto es, hacia los grupos de edad cada vez más avanzados. Este desplazamiento se produjo más tardíamente en la Comunidad Canaria que en el resto del país. Ya en la década del 2000, la creciente actividad es más visible entre las cohortes jóvenes y jóvenes-adultas, que a pesar de contraer matrimonio y dedicarse a la crianza y cuidado de los hijos han optado por no abandonar su puesto de trabajo (Cacouault, 2000. Durán; Serra y Torada, 2001. Maruani, 2002. Méda, 2002). Asimismo, este fenómeno también ha sido notable entre las cohortes de 35-39 y 40-44 años que, o bien no han dejado de

\footnotetext{
4 A finales del 2008, y debido a la actual crisis económica mundial, que ha afectado considerablemente al sector de la construcción, el número de parados ha llegado a superar al de paradas.
} 
trabajar, o han vuelto a incorporarse, o incluso se han integrado laboralmente por primera vez. En las últimas cuatro décadas el fenómeno de la reinserción laboral de las mujeres ha resultado ser más evidente entre las españolas que entre las canarias, aunque en los últimos años estas diferencias se han recortado, e incluso se han equiparado, especialmente entre las mujeres de las islas capitalinas y resto de las españolas.

En la década del 2000, la curva del empleo femenino de la comunidad canaria muestra ya prácticamente la misma descripción que la del resto del país (en forma de U invertida), y de otros países europeos, especialmente del mediterráneo (Grecia, Italia y Portugal), que han experimentado evoluciones similares a las de España (Borderías, 2003; Moreno, 2002; Moreno, 2005).

Algunos estudios han constatado que la generación española que marcó la nueva biografía laboral de las mujeres fue la nacida entre 1960-1964 (Garrido, 1993). En el caso de la comunidad canaria, el cambio de la actividad laboral de las mujeres fue protagonizado por la generación nacida entre 1967-1971. Esta cohorte empezó a beneficiarse de la enseñanza obligatoria y gratuita, y es la que comenzó a dedicarle más tiempo a la formación, manteniendo una actitud laboral más optimista y estable que sus progenitoras. En las últimas cuatro décadas, las madres y abuelas de las cohortes más jóvenes, fundamentalmente las que nacieron entre 1932-1936, son las que han alcanzado la máxima desigualdad ante la actividad y el empleo y, junto con la generación nacida entre 1927-1931, son las que han mantenido una máxima desigualdad frente al desempleo. Se trata de las canarias nacidas antes y durante la guerra civil española, con una reincorporación laboral casi inexistente, aunque, por supuesto, con una gran presencia en la economía no formal.

En los últimos años prácticamente ha desaparecido el carácter procíclico de la actividad y del empleo femenino, que en Canarias todavía era evidente hasta los 90. Ello fue protagonizado por las cohortes más jóvenes, y especialmente por las canarias más formadas que, por otro lado, son las que en menor medida sufren la situación de “desánimo laboral” y de "paro encubierto". Fenómenos que, dicho sea de paso, en las últimas décadas ha tendido a repercutir más negativamente sobre las canarias que sobre 
el resto de las españolas. No obstante, y especialmente a partir de mediados de la presente década, las canarias más formadas son las que en mayor medida están sufriendo el fenómeno de la sobrecualificación laboral (Alonso, 2001; Daune-Richard, 2000; Fernández y Jimeno, 2004).

En las cuatro últimas décadas el incremento de la actividad femenina se ha visto acompañado del retroceso de la actividad masculina. En la comunidad canaria este fenómeno se produjo más tardíamente que en el resto del país, además, no en todas las islas del Archipiélago este fenómeno se produjo con la misma intensidad. Hasta bien entrados los 90, en las islas más rurales del Archipiélago (El Hierro y La Gomera) el aumento de la población activa femenina no había sido tan notable como para contrarrestar el descenso de los activos. Si bien en la actualidad las mujeres de las islas rurales no pierden tanta actividad y empleo como en años anteriores, éstas suelen abandonar el trabajo (aunque no tanto el mercado laboral), para dedicarse a la reproducción y crianza de los hijos, con mayor intensidad que las mujeres de las islas capitalinas. Asimismo, el fenómeno de la reinserción laboral es más frecuente entre las mujeres de las islas capitalinas que entre las mujeres de las islas rurales, especialmente entre las mujeres mayores de 40-45 años.

A través de los procesos de desarrollo y modernización, el desempeño del trabajo a través de lo que se ha denominado "formas de producción doméstica", donde mayoritariamente las mujeres comenzaron trabajar, dio paso al trabajo asalariado. Entre las décadas de los 70 y hasta la actualidad, se ha producido una variación descendente de las/os empleadas/os en esta situación. Esta ha sido más considerable entre la población canaria que entre la española, y más especialmente entre el colectivo

\footnotetext{
${ }^{5}$ Consiste en aquellas actividades no remuneradas que son realizadas por y para los miembros del hogar. Actividades que pueden ser sustituidas por bienes y servicios pagados y obtenidos a través del mercado, tales como renta, condiciones de mercado e inclinaciones personales que permiten que el servicio fuera delegado a alguien ajeno al grupo familiar. Quedan incluidas dentro de la producción doméstica, las categorías de "ayudas familiares" y "los/as empresarios/as sin asalariados/as". Se denomina así, no porque el trabajo se desarrolle en el domicilio sino porque la retribución viene dada a través de la manutención y alojamiento.
} 
femenino que entre el masculino. No obstante hay que subrayar, que en los períodos de baja económica parece producirse un ligero aumento de la población ocupada en las "formas de producción doméstica". Parece que estos trabajos tienden a proteger el empleo en los momentos más críticos de la economía.

La inserción masiva de las mujeres al mercado de trabajo se produjo especialmente a través del sector público. La continua creación de empleo femenino a través de este sector, incluso en los momentos de baja económica, ha hecho posible que las mujeres no perdieran ocupación. Este sector ha servido de refugio al empleo femenino, además les ha ofrecido a las mujeres (en mejores condiciones que el privado) poder compaginar la maternidad y el cuidado de la familia con el trabajo extradoméstico. No ha de extrañar que las mujeres hayan utilizado como estrategias la mayor inversión en educación para poder ocupar los puestos laborales de este sector.

En las cuatro últimas décadas se puede apreciar, que la crisis económica incide más negativamente sobre la pérdida de la población asalariada que ocupada. Por el contrario, en las etapas de alza económica, la población ocupada crece menos que la asalariada. Especialmente ello se aprecia en las ramas de actividad más cíclicas: "agricultura", “construcción", “comercio", "hostelería”, “alimentación”, "bebidas” y "tabaco", con notable presencia femenina.

En la comunidad canaria, la incorporación masiva de las asalariadas se produjo especialmente a mediados de $\operatorname{los} 80$, años de plena inserción laboral. A finales de dicha década las canarias asalariadas superaban numéricamente a los asalariados, fenómeno que se produjo más tardíamente en el ámbito nacional. La mayoría de las mujeres que comenzaron a trabajar a través de este sector se incorporaron a él de forma directa. Esto es, la creación de empleo no fue tan considerable para las mujeres que ya trabajaban fuera del hogar, como sí para las generaciones que directamente se integraron en el mercado de trabajo. Principalmente se trata de las hijas y nietas de las cohortes que durante el proceso de tercerización económica habían tenido que abandonar su empleo en el sector agrario, viéndose obligadas a trabajar exclusivamente en el hogar o en la economía sumergida. 
En la segunda mitad de la década del 2000, tres cuartas partes de las canarias empleadas en el sector agrario mantienen una situación profesional como asalariadas del sector privado. El resto de las empleadas en la agricultura trabajaban como autónomas y en ayuda familiar. En el ámbito estatal, la mayoría de las ocupadas en la agricultura constan como autónomas, seguidas de la ayuda familiar y asalariadas del sector privado.

Las canarias de la industria trabajan principalmente en el sector privado, seguidas de las autónomas, ayuda familiar, asalariadas del sector público, empleadoras y en cooperativas. Las españolas trabajadoras de este sector, principalmente constan como asalariadas del sector público.

La mayoría de las canarias y españolas de la construcción trabajan en el sector privado y en situación de ayuda familiar.

Más de la mitad de las canarias y españolas de servicios son asalariadas del sector privado. Las canarias autónomas y en cooperativas de este sector se sitúan por debajo de las españolas. Como empleadoras y en ayuda familiar la situación entre las canarias y el resto de las españolas es más parecida.

En todas las islas del archipiélago canario las mujeres trabajan principalmente como asalariadas, aunque el empleo en las "formas de producción doméstica" tiende a incrementarse en las islas más rurales. Así, La Gomera tiene el mayor número de mujeres trabajando en situación de ayuda familiar, y El Hierro el mayor número de autónomas, y principalmente trabajan en el sector agrario. No obstante, a pesar de tratarse de las islas más rurales no por ello cuentan con más asalariadas en la agricultura. Este fenómeno también deja entrever la situación de las mujeres que, a pesar de desarrollar estas actividades, no se declaran como ocupadas, ni siquiera en situación de ayuda familiar, aunque este fenómeno es más visible entre las mujeres mayores que entre las jóvenes y jóvenes-adultas.

Por otro lado podemos observar que en los últimos 40 años la segregación laboral entre los géneros se ha reflejado con toda claridad en la sobre-representación y sub-representación sectorial, profesional y ocupacional femenina (Casal, 2000; Rubery, 
1993). Así, y a pesar del paulatino descenso de las canarias empleadas en el sector agrario, en la actualidad éste se trata de un sector con un alto nivel de integración.

Durante las últimas cuatro décadas, las canarias no han dejado de estar sobrerepresentadas en el conjunto del sector servicios. En el sector industrial las tasas de feminización se han situado muy por debajo de la tasa global. En el sector construcción la sub-representación de las canarias ha sido más que evidente.

Tanto las canarias como las españolas mantienen sobre-representación en las ramas de "servicio doméstico", "saneamiento", "servicios personales", "limpieza", “asistencia social”, "hostelería”, "sanidad” y “educación”. También en algunas ramas de la industria, como en "confección” y "alimentación”.

En los últimos años ha desaparecido la sub-representación de las mujeres en algunas de las ramas del sector servicios, incluso en algunas de ellas se ha producido sobre-representación, tales como en la "administración pública" e "instituciones financieras", "actividades auxiliares a la intermediación financiera, seguros y planes de pensiones", “actividades inmobiliarias", “investigación”, “desarrollo”, “otras actividades empresariales" y "actividades recreativas y culturales".

La sub-representación de las mujeres es bastante clara en la totalidad de las ramas del sector industrial y construcción.

Históricamente las mujeres han permanecido excluidas de ciertas ramas, tal es el caso de "construcción de máquinas" y "material mecánico", "construcción de máquinas y material de oficinas y ordenadores", “construcción de máquinas y material electrónico", “fabricación/reparación de material electrónico, equipos, aparatos de radio, T.V.”, etc. En los últimos años se observa la presencia de mujeres en algunas de estas ramas, pero ésta aún es exigua, con una mayor importancia cualitativa que cuantitativa, y menos significativa en Canarias que en ámbito nacional.

En cuanto a la situación profesional, la sub-representación como "empleadoras" es evidente en todos los sectores económicos. Esta sólo desciende en el sector servicios, donde aún así éstas representan menos de una cuarta parte en comparación con los empleadores. 
En todos los sectores también se produce segregación profesional de las “autónomas", aunque la sub-representación parece disminuir en el sector servicios, y menos entre las canarias que entre el resto de las españolas.

La sub-representación de las mujeres también tiene lugar en la situación profesional de "cooperativas".

La segregación profesional es evidente en "ayuda familiar", aunque esta se caracteriza más por la sobre-representación. La mayoría de las mujeres que trabajan en esta situación desarrollan su trabajo en los sectores de la agricultura y servicios.

La tasa de feminización ${ }^{6}$ de las canarias y españolas asalariadas en servicios se sitúa por encima de la tasa global de feminización. Entre las asalariadas de servicios se puede decir que existe integración.

A medida que se incrementan las mejoras de las condiciones de la situación profesional (valoradas en términos de estatus laboral, social y de remuneración), la representación femenina disminuye. Por el contrario, a medida que desciende el nivel de estas condiciones la presencia de las mujeres se hace mayor (Wirth, 2002). La segregación también es evidente en la situación ocupacional, que se caracteriza por una sobre-representación y sub-representación en los diferentes tipos de profesiones.

Hasta bien entrada la década del 2000, el colectivo de "dirección de las empresas y de la administración pública y empresas privadas y públicas” presentan una tasa de feminización por debajo de la tasa global. Incluso entre las mujeres más jóvenes, actualmente más formadas que los varones, la presencia femenina es inferior a la masculina. Todavía se produce sub-representación femenina en estos tipos de ocupaciones, caracterizadas por importante responsabilidad, elevados salarios y alto nivel de estatus social.

Entre los "técnicos y profesionales científicos e intelectuales", la tasa de feminización se situaba por encima de la tasa global de feminización, fenómeno que resulta más apreciable en la comunidad canaria que en el Estado. Este grupo, donde

\footnotetext{
${ }^{6}$ Porcentaje de mujeres empleadas en cada sector sobre el total del empleo.
} 
están incluidos los titulados superiores, medios y otros tipos de profesiones, se caracteriza por su alta remuneración y prestigio social. Tanto en Canarias como en el ámbito nacional se produce una sobre-representación femenina, que obedece al elevado número de mujeres ocupadas en "educación”, “sanidad”, y a la asalarización femenina en el sector público.

Entre el colectivo de "técnicos y profesionales de apoyo", la tasa de feminización de las canarias se sitúa por debajo de esta tasa global, mientras que la de las españolas se coloca por encima. Por ello, en Canarias en este grupo de profesionales tiene lugar una sub-representación femenina, mientras que en el Estado se produce una integración de las mujeres, por lo menos en el conjunto de estas profesiones.

En el ámbito nacional, el grupo de "personal administrativo" tiene la mayor tasa de feminización. En Canarias, después de las "técnicos y profesionales científicos e intelectuales", este colectivo condensa la segunda tasa de feminización, por ello se produce sobre-representación, lo que principalmente ha obedecido al incremento de la asalarización en el sector público. La sobre-representación de las mujeres en estas profesiones ha estado determinada por el peso de las cohortes jóvenes y jóvenes-adultas ocupadas en puestos administrativos.

El colectivo de "trabajadores de los servicios de restauración, personales, protección y vendedores de los comercios" se caracteriza por su elevada feminización, esto es, por una sobre-representación de las mujeres. En el caso de Canarias, este grupo está tan feminizado como el grupo de las "trabajadoras no cualificadas", profesiones que se caracterizan por su menor categoría social y económica.

La tasa de feminización en el grupo de los "trabajadores cualificados en la agricultura y pesca" se sitúa por debajo de la tasa global de feminización. Por ello se produce sub-representación femenina, además, esta es más visible entre las canarias que entre el resto de las españolas.

Mayor es aún la segregación ocupacional en el grupo de "artesanos y trabajadores cualificados en la industria manufacturera, construcción y minería”, así como entre los “operadores de instalaciones, maquinaria y montadores". La sub- 
representación de las mujeres en estos colectivos profesionales es mayor en Canarias que en el ámbito nacional.

Se observa igualmente, que el tipo de "segregación horizontal" (profesiones y categorías) es menos acentuada que la "segregación vertical" (jerárquica). En general, las canarias mantienen una menor relación que el resto de las españolas con las ocupaciones que exigen de un alto y medio nivel tecnológico.

Ante la mayor participación laboral de las mujeres y las nuevas estructuras relacionadas con los puestos de trabajo, no resulta tan obvio el estancamiento de la división sexual del trabajo, más aún, parece que se ha producido una reestructuración de las pautas de segregación del mismo (Borderías, 2005). Debido a ello, la competencia por los puestos de trabajo parece ser más obvia entre las mujeres de las diversas generaciones que entre los géneros.

\subsection{Formación para el (des) empleo}

En las últimas cuatro décadas ha existido una cierta correlación "positiva" entre el nivel de estudios de las mujeres y su relación con la actividad económica. Esto es, en términos generales se aprecia que a medida que aumentan los niveles de formación de las canarias, su relación con el mercado de trabajo se hace mayor y, viceversa, a medida que disminuye el nivel de formación de las mujeres su relación con el trabajo extradoméstico decrece. Entrada la presente década, si bien esta correlación todavía es evidente, nos encontramos con muchas mujeres que, incluso con altos niveles de formación, se ven obligadas a trabajar en otras formas de la economía, especialmente en la economía no formal. En el caso de otras mujeres, especialmente inmigrantes, ello también es evidente en la economía ilícita (Cachón, 2003; Ybarra, Hurtado y San Miguel, 2001).

Durante los últimos cuarenta años las canarias y españolas en situación de actividad y empleo con estudios secundarios y universitarios ha tendido a situarse por encima de la tasa media de la actividad y ocupación femenina. Por el contrario, las 
activas y ocupadas con bajos niveles de estudios (estudios primarios y de EGB-bachiller elemental) han tendido a situarse por debajo de la media de la actividad y empleo femenino.

En términos generales, cuanto menos tiempo le han dedicado las mujeres a la formación más pronto han comenzado a trabajar, pero también antes y en mayor medida se producía su retirada laboral (debido al matrimonio y a la crianza de los hijos). Contrariamente, cuando más tiempo han invertido las mujeres en los estudios más tarde empezaban a trabajar, descendiendo las posibilidades de abandonar su puesto de trabajo. Por ello, las mujeres que más han retrasado su inserción laboral son las universitarias, aunque en comparación éstas son las que más pronto tienden a encontrar trabajo. También son las que menos abandonan el empleo para dedicarse al cuidado de la familia y al trabajo del hogar. Es por ello que, hasta finales de los 90, la máxima participación laboral de las canarias con estudios primarios y de EGB-bachiller elemental se producía entre los 20-24 años, mientras que en la década del 2000 se ha extendido a 25-29 años. Las mujeres con estudios secundarios mantenían, hasta principios del 2000, una ocupación máxima entre los 25-29 años. Actualmente ésta se ha extendido a 30-34 años, y sostienen altos índices de empleo a edades más avanzadas. Hasta entrada la presente década, las universitarias mantenían la máxima participación laboral hasta los 34 años. En los últimos años ésta se ha prolongado hasta los 39 años, y muchas de estas mujeres no abandonan el mercado laboral.

En la actualidad, el comportamiento laboral que tienen las mujeres canarias con estudios universitarios se asemeja al que tenían las mujeres con estudios secundarios en la década de los 70, ya que éste era el máximo nivel de estudios alcanzado por el colectivo femenino. Parece ser que, en las últimas cuatro décadas, la formación de las mujeres ha evolucionado de manera más rápida que su comportamiento laboral. Independientemente del fenómeno de integración laboral y del evidente rechazo de las cohortes más jóvenes por no abandonar el mercado de trabajo en las últimas décadas, habría que destacar el hecho de la integración y reintegración laboral de las mujeres menos jóvenes y con escasos niveles de estudios. 
Hasta mediados de los 80, las paradas sin experiencia de la comunidad canaria contaban principalmente con estudios primarios. En la actualidad, la mayoría de las paradas sin experiencia laboral tienen estudios de EGB-bachiller elemental. Debido a la creciente competitividad laboral entre las cohortes generacionales, la presencia de estas paradas ha tendido a prolongarse en el tiempo, sufriendo las consecuencias de paro de larga duración. En general, la tónica observada en el comportamiento de las paradas de la comunidad canaria durante las cuatro últimas décadas, es que las más formadas padecen menos las consecuencias del paro inexperto y exptero que las mujeres menos formadas.

En los últimos años, las mujeres con estudios primarios y de EGB-bachiller elemental son las que más han perdido empleo. Ello ha respondido a la competitividad laboral con las mujeres más formadas, especialmente con las que tienen estudios secundarios, ya que éstas tienden a estar más dispuestas que las universitarias a aceptar los trabajos que en años anteriores realizaban las mujeres con el bachiller elemental.

A las mujeres les resulta más difícil encontrar empleo que a los varones. Sólo entre el colectivo de los jóvenes (16-19 años), los parados sin experiencia aventajan numéricamente a las paradas inexpertas. Ello es debido a que los varones comienzan a trabajar antes que las mujeres, ya que ellas continúan estudiando. A partir de los 30 años, las paradas que no han trabajado antes superan numéricamente a los parados, situación que se agrava a medida que desciende el nivel de estudios.

\subsection{Formación y trabajo: cambios en el calendario matrimonial}

A lo largo de las cuatro últimas décadas, la mayor inversión en formación también ha condicionado otra serie de transformaciones en la vida de las mujeres, principalmente ha trastocado su situación familiar y con ello algunas de las circunstancias que históricamente han condicionado e imposibilitado su inserción en el mercado de trabajo. Entre otra serie de factores, estos cambios se han expresado: en el incremento de la soltería, posposición en la edad de entrada al matrimonio, y en las 
mayores posibilidades de la disolución del mismo. Así, desde la década de los 70, las mujeres han retrasaron la edad de entrada al matrimonio en 6 años. En la década de los 70 las canarias y españolas contraían matrimonio a los 22 y 25 años respectivamente. Actualmente, las canarias se casan sobre los 29 años, y el resto de las españolas a los 31 años. En los últimos 40 años, las canarias han tendido a casarse antes que el resto de las españolas.

Entre las cohortes más jóvenes la posibilidad de nupcialidad es más prolongada en el tiempo que entre las generaciones anteriores, que o bien solían contraer matrimonio a unas determinadas edades o permanecían solteras el resto de sus vidas. En el Archipiélago Canario, el fenómeno de la prolongación de la soltería comenzó a producirse con anterioridad en las islas capitalinas. Si bien en todas las Islas, las mujeres están invirtiendo más tiempo en formación, más incluso que sus coetáneos varones, las mujeres de las islas rurales se casan antes que las mujeres de las islas capitalinas.

A finales de los 70, las españolas comenzaron a retrasar la edad de entrada al matrimonio, elevándose la edad de las madres españolas. En Canarias, este fenómeno tuvo lugar años más tarde, en la segunda mitad de los 80. Desde la década de los 70, la edad media de las canarias al nacimiento de sus hijos no ha dejado de situarse por debajo de la media española aunque en los últimos años estas diferencias se han recortado. A principios de la presente década, la edad media de las españolas y de las canarias en tener a sus hijos se situaba entre los 32-33 años y 30-31 años respectivamente, aunque en las islas más rurales esta media descendía a los 28 años.

Las canarias nacidas a finales de los 60 y principios de los 70, que fueron las precursoras de los nuevos cambios formativos y laborales, han sido también las iniciadoras en retrasar la edad de tener a sus hijos. A principios del 2000, sus maternidades se comenzaron a unir con las de las mujeres menores de 30 años, que también la habían retrasado, debido a la mayor inversión de tiempo en los estudios y a su mayor participación laboral.

Por otro lado podríamos subrayar, que si bien en algunos casos la familia compleja ha podido facilitar la salida de las otras mujeres del ámbito doméstico (unas 
mujeres se hacían cargo del trabajo del hogar y cuidado de la familia y otras salían a trabajar), en general la atención y el cuidado de las personas dependientes ha recaído sobre la figura de la madre-esposa. Ha de suponerse que, debido a las mayores exigencias por parte de sus miembros, el sometimiento de las mujeres a las tareas domésticas es superior en los hogares extensos y múltiples, que dicho sea de paso han sido, y aún son, más comunes en la comunidad canaria que en resto del Estado. Este ha sido otro de los factores que ha explicado las menores posibilidades que tuvieron las canarias, frente al resto de las españolas, ante la inserción laboral.

A pesar de los cambios experimentados en las pautas laborales de las mujeres, éstas continúan siendo las encargadas, prácticamente en su exclusividad, de la realización del trabajo doméstico (Papí y Frau, 2005; Tobío, 2002 y 2005; Torns, 2003). A principios de los 90, entre algunos colectivos femeninos, especialmente las más jóvenes, ya se apreciaba el claro rechazo que mantenían las canarias a declararse "amas de casa".

El cambio de rol económico de las mujeres no sólo ha sido anterior a los cambios observados en la institución familiar (posposición de soltería, retraso en la edad de entrada al matrimonio, mayores posibilidades de separación y divorcio, etc.), sino que también éste ha sido anterior a las pautas de conducta experimentadas dentro del grupo doméstico. Esto es, en las últimas décadas los cambios producidos en las pautas de las mujeres han fluido más desde los espacios exteriores (esfera extradoméstica) a los interiores (esfera doméstica), que viceversa. Las mujeres continúan realizando prácticamente en su exclusividad el trabajo doméstico, el cuidado y la atención a la familia.

La expulsión de las mujeres del mercado de trabajo en las etapas de baja económica, más especialmente en la década de los 70 y primeros años de los 80 , se vio reflejada en el incremento de su declaración como "amas de casa". En los últimos años, y debido a que cada vez más las mujeres se niegan a retirarse el mercado de trabajo, este fenómeno ha perdido fuerza, especialmente entre las jóvenes y jóvenes-adultas. Esto es, las mujeres prefieren constar más como desempleadas, que declararse trabajadoras del 
hogar (amas de casa). En Canarias, la generación que marcó este cambio de actitud hacia el trabajo del hogar fue la nacida entre 1967-1971. La dedicación exclusiva al empleo y a la doble jornada se ha producido especialmente entre las mujeres de 21-45 años.

En los momentos de baja económica, muchas mujeres que trabajan en la economía formal son expulsadas del mercado, viéndose obligadas a trabajar exclusivamente en el hogar. Otras empleadas en la economía formal pasan a trabajar a la economía no formal. Otras mujeres dedicadas exclusivamente al trabajo del hogar (y debido a la situación de desempleo de sus cónyuges y otros miembros del hogar) se ven obligadas a trabar fuera, especialmente en la economía no formal. No obstante, todas ellas están obligadas a trabajar en los hogares. La fluctuación del colectivo de una esfera a otra resulta obvia. A pesar del mayor nivel formativo de las mujeres en dichos ámbitos, éstas continúan desempeñando las mismas actividades que han realizado a lo largo de la historia, y que podrían resumirse en: servir, cuidar y educar. Trabajos que, en general, están dispuestas a realizar en las condiciones que sea. Sin embargo, y de forma contradictoria, todavía aún se sigue clasificando a una parte del colectivo femenino como improductivo (Arrazola, 2001).

\section{Conclusiones}

A lo largo de la historia las mujeres han desempañado trabajos extradomésticos, pero esta situación no ha sido reconocida por las estadísticas del empleo, ni siquiera en la categoría de "ayuda familiar". En el ámbito nacional y en la comunidad canaria, tenemos que esperar hasta la década de los 60 y 70 respectivamente, para observar cómo estadísticamente las mujeres trabajan fuera del ámbito doméstico. Concretamente, a nivel del Estado, la generación que marcó la nueva biografía laboral de las mujeres fue la nacida entre 1960-1964. En el caso de la comunidad canaria, el cambio de la actividad laboral de las mujeres fue protagonizado por la generación 
nacida entre 1967-1971. Esta cohorte empezó a beneficiarse de la enseñanza obligatoria y gratuita, y es la que comenzó a dedicarle más tiempo a la formación, manteniendo una actitud laboral más optimista y estable que sus progenitoras.

Hemos podido ver que, pese a las peculiaridades sociodemográficas, económicas y culturales que presenta el archipiélago canario, los procesos de cambio social experimentados en las últimas cuatro décadas han afectado a las canarias de forma similar que al resto de las españolas. Las mujeres han tendido a trabajar fuera de los hogares principalmente por necesidades económicas, sin embargo, especialmente a principios de la década de los 90, se aprecia una mayor persistencia de las mujeres canarias por encontrar trabajo y por no perderlo una vez que lo consiguen. En parte es síntoma de que cada vez más las mujeres tienen a trabajar por una decisión voluntaria, y, posiblemente, emancipadora. Ello ha sido evidente entre las cohortes más jóvenes y entre las más formadas. Debido a la creciente reinserción de las mujeres y a la imitación de las pautas laborales por parte del resto de los colectivos ello también se ha hecho visible entre las adultas (30-44 años) y mayores (45-59 años).

A pesar de los cambios sustanciales que ha experimentado el mercado de trabajo en las últimas cuatro décadas, la creciente inversión en formación por parte de las mujeres ha posibilitado su mayor relación con el empleo, así como una mejora de su estatus en lo que respecta a su mayor autonomía y posición social. Resta que esta posición sea en condiciones de igualdad. Concluye aquí pues, una indagación escasamente explorada en las investigaciones españolas sobre la situación laboral de las mujeres canarias. 


\section{BIBLIOGRAFÍA}

- Aguilar, M. (2001): La inserción laboral de los jóvenes en España. Un enfoque microeconométrico. Málaga: Tesis Doctoral. Universidad de Málaga.

- Alberdi, I, Escario, P. y Matas, N. (2002): Las mujeres jóvenes en España, colección estudios sociales. $\mathrm{N}^{\mathrm{o}}$ 4. Barcelona: Fundación la Caixa.

- Alonso, L. (2001): Trabajo y posmodernidad: el empleo débil. Madrid: Fundamentos.

- Arrazola, M. (2001): Análisis empírico de la depreciación del capital humano para el caso de las mujeres y los hombres en España. Madrid: Instituto de Estudios Fiscales.

- Ayllon, Ma.D. (1995): "Integración de la mujer en la vida laboral en la actual coyuntura socioeconómica”. En Carrasco, M; García, A; Labrador, J y Alemany, C. (eds). Mujer, trabajo y maternidad. Madrid: U.P.C.O.

- Barrera, Ma.C. (2001): Las mujeres herreñas en cifras. Dos décadas: 1977-2001. Tenerife: Cabildo Insular de El Hierro.

- (2004): Trabajo, educación y familia de las mujeres canarias. Análisis sociológico del papel de las mujeres en los últimos 20 años. Tesis doctoral (inéd.). La Laguna: Universidad de La Laguna.

. (2004): "Panorama laboral de las canarias. Décadas 70-90”. En Tebeto. Anuario del Archivo Histórico Insular de Fuerteventura, nº 16, pp. 313-330.

. (2005): "Las diferencias de género en el sistema educativo y sus incidencias en el acceso al empleo". En Bordón, no 57 (4), pp.113-125.

. (2007): Mujeres y cambio social en Canarias. Oviedo: KRK.

- Beck-Gersheim, E., Butler, J. y Puigvert, L. (2001): Mujeres y transformaciones sociales. Barcelona: El Roure.

- Benerías, L. (2005): “La globalización y el trabajo de las mujeres”. En R. Antúnez, Los sentidos del trabajo. Buenos Aires: Antítesis. 
- Borderías, C. (2003): "la feminización de los estudios sobre el trabajo de las mujeres: España en el contexto internacional (1969-2002)”. En Sociología del Trabajo, no 48, pp. 57-124.

- Cachón, L. (2000): La inserción profesional. Alzira: Germania.

. (2003): Inmigración y segmentación de los mercados de trabajo en España. Sevilla: Centra, Documento de trabajo S2003/02.

- Cacouault, M. (2000): "Variaciones en los empleos femeninos y masculinos". En Maruani, M.; Rogerat, C. y Torns, T. (dirs.) Las nuevas fronteras de la desigualdad. Hombres y mujeres en el mercado de trabajo. Barcelona: Icaria, pp.27-34.

- Carrasco, C. y Mayordomo, M. (2000): "Los modelos y estadísticas de empleo como construcción social. La encuesta de población activa y el sesgo de género”. En Política y Sociedad, no 34, pp. 101-112.

- Casal Bataller, J. (2000): "Modalidades de transición profesional y precarización del empleo". En Cachón, L. (ed.) La inserción profesional. Alzira: Germania, pp. 17-54.

- Castaño, C. et al (2004): Indicadores laborales básicos de la situación de la mujer en España y sus regiones. Madrid: Instituto de la Mujer.

- Daune-Richard, A-M. (2000): “Cualificación y representación social”. En Maruani, M. et al (dirs.) Las nuevas fronteras de la desigualdad. Hombres y mujeres en el mercado de trabajo. Barcelona: Icaria, pp. 71-88.

- Durán, Mª.A. (1992): El trabajo de la mujer en España. Un estudio sociológico. Madrid: Tecnos.

- Durán, Ma.A. Serra, I. y Torada, R. (2001): Mujer y trabajo. Problemática actual. Valencia: Germania.

- Garrido, L. (1993): Las dos biografías de la mujer en España. Madrid: Instituto de la Mujer. Ministerio de Asuntos Sociales. Madrid.

- Ibánez, M. (1998): Género y familia en la inserción laboral. Oviedo: Servicio de Publicaciones de la Universidad de Oviedo.

- Maruani, M. (2002): Trabajo y el empleo de las mujeres. Madrid: Fundamentos.

- Méda, D. (2002): El tiempo de las mujeres. Conciliación entre vida familiar y profesional de hombres y mujeres. Madrid: Narcea. 
- Montañes, M. y Olivari, L. (2000): Jóvenes y Empleo. Percepciones, valores y expectativas. Madrid: GPS.

- Moreno, A. (2005): “Empleo de la mujer en los regímenes de bienestar del sur de Europa en perspectiva comparada. Permanencia del modelo de varón sustentador". En REIS, nº 112, pp. 131-163.

- Moreno, L. (2002): Bienestar mediterráneo y “Supermujeres”. En RES, n 2, pp. 4157.

- Mosteiro, M.J et al (2001): "Modelos y factores que influyen en el desarrollo de la carrera de las mujeres”. En R. Rald Philipp. Cuestiones actuales de la Sociología del Genero. Madrid: CIS, pp. 105-125.

- Papí, N. y Frau, M.J. (2005): "La conciliación del empleo y del hogar: respuesta y reflejo de una organización del trabajo construida desde la institución de género". En REIS, no 110 , pp. 149-171.

- Segura, J. (2001): "La reforma del mercado de trabajo español: un panorama". En Revista de Economía Aplicada, nº 9, pp. 157-90.

- Suárez, I. (2004): Mujeres canarias/cambio social. Gran Canaria: Colectivo de Mujeres Canarias.

- Tobío, C. (2002): “Conciliación o contradicción: cómo hacen las madres trabajadoras". En REIS, nº 97, pp. 155-186. . (2005): Madres que trabajan. Dilemas y estrategias. Madrid: Cátedra.

- Torns, T. (2000): "Las mujeres jóvenes en el mercado de trabajo en España". En Cachón, L. Juventudes y Empleo: perspectivas comparadas. Madrid: INJUVE. . Torns, T. et al (2003): "La conciliación de la vida laboral y familiar ¿un horizonte posible?”. En Sociología del Trabajo, nº 50, pp. 111-153.

. Torns, T. (2005): "De la imposible conciliación a los permanentes malos arreglos". En Cuadernos de Relaciones Laborales Vol. 23, n 1, pp. 15-33.

- Ybarra, J. A.; Hurtado, J., y Miguel, B. (2001): "La economía sumergida revisitada". En Sociología del Trabajo, nº 43, pp. 29-70. 